\title{
Solar Orbiter Radio and Plasma Waves - Time Domain Sampler: In-flight performance and first results
}

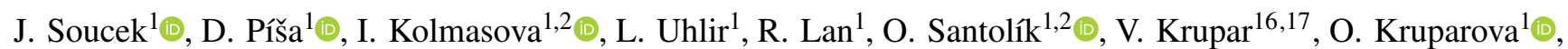
J. Baše ${ }^{1}$, M. Maksimovic ${ }^{3}$, S. D. Bale ${ }^{4,5}$, T. Chust $^{6} \odot$, Yu. V. Khotyaintsev ${ }^{7} \odot$, V. Krasnoselskikh ${ }^{8}$, M. Kretzschmar ${ }^{8,9}$ (], E. Lorfèvre ${ }^{10}$, D. Plettemeier ${ }^{11}$, M. Steller ${ }^{12}, \check{S}_{\text {. Štverák }}^{13,1}$, A. Vaivads ${ }^{7,14}$, A. Vecchio ${ }^{3,15}$, D. Bérard ${ }^{3}$, and X. Bonnin ${ }^{3}$

${ }^{1}$ Institute of Atmospheric Physics, Czech Academy of Sciences, Boční II/1401, 14100 Prague, Czech Republic e-mail: soucek@ufa.cas.cz

2 Faculty of Mathematics and Physics, Charles University, V Holesovickach 2, 18000 Prague 8, Czech Republic

3 LESIA, Observatoire de Paris, Université PSL, CNRS, Sorbonne Université, Université de Paris, 5 place Jules Janssen, 92195 Meudon, France

4 Space Sciences Laboratory, University of California, Berkeley, CA, USA

5 Physics Department, University of California, Berkeley, CA, USA

${ }^{6}$ LPP, CNRS, Ecole Polytechnique, Sorbonne Université, Observatoire de Paris, Université Paris-Saclay, Palaiseau, Paris, France

7 Swedish Institute of Space Physics (IRF), Uppsala, Sweden

8 LPC2E, CNRS, 3A Avenue de la Recherche Scientifique, Orléans, France

9 Université d'Orléans, Orléans, France

10 CNES, 18 Avenue Edouard Belin, 31400 Toulouse, France

11 Technische Universität Dresden, Würzburger Str. 35, 01187 Dresden, Germany

12 Space Research Institute, Austrian Academy of Sciences, Graz, Austria

13 Astronomical Institute of the Czech Academy of Sciences, Prague, Czech Republic

14 Department of Space and Plasma Physics, School of Electrical Engineering and Computer Science, Royal Institute of Technology, Stockholm, Sweden

15 Radboud Radio Lab., Department of Astrophysics, Radboud University, Nijmegen, The Netherlands

16 Goddard Planetary Heliophysics Institute, University of Maryland, Baltimore County, Baltimore, MD 21250, USA

17 Heliospheric Physics Laboratory, Heliophysics Division, NASA Goddard Space Flight Center, Greenbelt, MD 20771, USA

Received 31 March 2021 / Accepted 14 June 2021

\section{ABSTRACT}

Context. The Radio and Plasma Waves (RPW) instrument on board Solar Orbiter has been operating nearly continuously since the launch in February 2020. The Time Domain Sampler (TDS) receiver of the RPW instrument is dedicated to waveform measurements of plasma waves and dust impact signatures in an intermediate frequency range from 0.2 to $200 \mathrm{kHz}$.

Aims. This article presents the first data from the RPW-TDS receiver and discusses the in-flight performance of the instrument and, in particular, the on-board wave and dust detection algorithm. We present the TDS data products and its scientific operation. We demonstrate the content of the dataset on several examples. In particular, we study the distribution of solar Langmuir waves in the first year of observations and one Type III burst event.

Methods. The on-board detection algorithm is described in detail in this article and classifies the observed waveform snapshots, identifying plasma waves and dust impacts based on the ratio of their maximum amplitude to their median and on the spectral bandwidth. The algorithm allows TDS to downlink the most scientifically relevant waveforms and to perform an on-board statistical characterization of the processed data.

Results. The detection algorithm of TDS is shown to perform very well in its detection of plasma waves and dust impacts with a high accuracy. The initial analysis of statistical data returned by TDS shows that sporadic Langmuir waves that are not associated with Type III events are routinely observed in the inner heliosphere, with a clear increase in occurrence rate closer to the Sun. We also present an example of RPW observations during an encounter of the source region of a Type III burst, which exploits the on-board calculated histograms data.

Key words. plasmas - solar wind - waves - Sun: radio radiation - space vehicles: instruments - Sun: heliosphere

\section{Introduction}

The Radio and Plasma Waves (RPW) instrument (Maksimovic et al. 2020) on Solar Orbiter includes three receivers for the digitization and on-board processing of electromagnetic field data across different frequency bands: The Low Frequency Receiver (LFR), covering the frequency range from well below $1 \mathrm{~Hz}$ to $10 \mathrm{kHz}$ with both time series data and spectral data; the Thermal Noise and High Frequency Receiver (TNR/HFR), providing electromagnetic spectra from several $\mathrm{kHz}$ to $16 \mathrm{MHz}$; and the Time Domain Sampler (TDS), a medium frequency receiver dedicated to waveform measurements. Specifically, TDS is designed to capture electromagnetic waveform snapshots in the frequency range from $200 \mathrm{~Hz}$ to $200 \mathrm{kHz}$, resolving, in 
particular, plasma waves near the electron plasma frequency and in the range between the proton and electron plasma frequencies, as well as transient signals in antenna voltage, associated with interplanetary dust impacts. In this article, we describe the operation of the TDS instrument and its data products. We also present an overview of the measurements obtained during its first year of operation, covering the commissioning phase and the first part of Solar Orbiter cruise phase (Müller et al. 2020). All the RPW data, including documentation, are available in the public Solar Orbiter Archive ${ }^{1}$. As per the Solar Orbiter data access rules, the data become public after three months from the time they are received on the ground.

One of the key science objectives of the RPW instrument is to improve our understanding of plasma instabilities in the solar wind, as well as the generation of high frequency plasma waves and their propagation and occurrence in the inner heliosphere (for a review see Gurnett 1991). The key measurements for this investigation are provided by the TDS receiver and include observations of Langmuir waves associated with interplanetary shocks, Type III radio bursts, and (at other times) less obvious source processes.

The RPW instrument measures intense Langmuir-like (Langmuir waves, beam mode Gary 1985 or Z-mode Malaspina $\&$ Ergun 2008) waves in the solar wind in association with suprathermal electron beams produced by either solar flares (e.g., Lin et al. 1981) or accelerated by interplanetary shocks (Bale et al. 1999). These waves are believed to undergo linear mode conversion or nonlinear wave-wave interactions that produce electromagnetic emissions at the local electron plasma frequency and its second harmonic (Ginzburg \& Zhelezniakov 1958; Gurnett \& Anderson 1976; Cairns \& Melrose 1985; Kellogg 1980; Kim et al. 2007). The process responsible for the conversion of largely electrostatic Langmuir waves to a radio emission is currently not fully understood and RPW measurements, together with data from other Solar Orbiter instruments, should help us improve our understanding of this process. In particular, the capability of TDS to measure one component of magnetic field up to $200 \mathrm{kHz}$ could provide unique novel measurements of the magnetic component of $Z$-mode waves and allow us to track the mode conversion process.

While numerous interplanetary shocks were observed by previous spacecraft, high frequency wave measurements are limited for those events and only a few good in-situ observations of the Type II source regions exist (Graham \& Cairns 2015); RPW specifically targets the interplanetary shocks and their foreshocks and it uses a dedicated triggered burst mode to obtain very high resolution measurements of those phenomena. TDS supports this mode by providing waveform snapshots with a very high cadence to characterize plasma waves associated with the shock and in particular the Langmuir-like waves in its foreshock.

RPW also encounters and detects other wave phenomena, such as plasma waves associated with magnetic holes (Lin et al. 1996; Briand et al. 2010), stream interaction regions, and other plasma discontinuities. In addition to the Langmuir waves discussed above, TDS also routinely observes ion-acoustic waves Doppler-shifted to kilohertz frequencies (see Píša et al. 2021, for an analysis of those waves). The RPW antennas also act a dust detector. Dust impacts on the spacecraft body result in the release of an electron cloud which is observed as a sharp transient spike in the voltage between RPW antennas and the spacecraft body 1 The Solar Orbiter Archive can be found at http://soar.esac.
esa.int
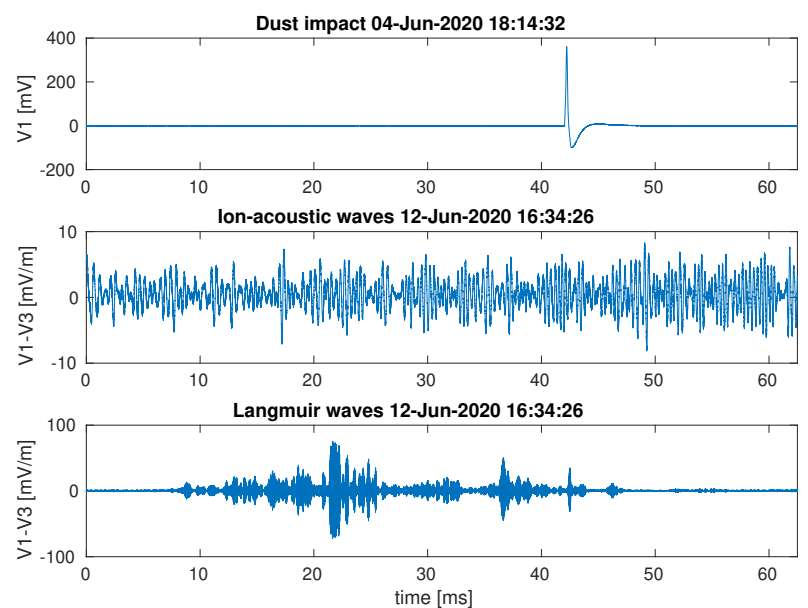

Fig. 1. Examples of typical triggered waveform snapshots from TDS Top: dust impact voltage spike. Middle: ion-acoustic wave with a frequency around $1 \mathrm{kHz}$. Bottom: Langmuir wave packets at the plasma frequency. All data shown in the figure are sampled at $262.1 \mathrm{ksps}$ and entire TDS snapshots of 16384 samples are presented.

(Meyer-Vernet et al. 2009). These events are recorded by TDS and can be detected and counted by the TDS receiver (Zaslavsky et al. 2021).

Figure 1 shows the three typical phenomena discussed above as observed by TDS in the first year of its operation. The top panel shows a typical dust impact voltage spike, indicating that a cloud of electrons from an evaporated dust particle impacting on the spacecraft briefly changed the spacecraft or antenna potential (see Zaslavsky et al. 2021, for a detailed discussion of the dust impacts observed on TDS). The middle panel shows an example of a measurement of an ion-acoustic wave. The waves have much lower frequencies in the plasma rest frame, but due to their short wavelengths, they are often Doppler shifted to kilohertz frequencies by the solar wind flow. In this case, the center frequency is approximately $1 \mathrm{kHz}$. The last panel shows an example of a snapshot containing several intense Langmuir wave packets.

We note that the dust impact in the top panel is plotted as antenna voltage, while the waves in the lower two panels as electric field, respecting the nature of the phenomena. The dust impact signature is a transient change in the voltage between the antenna and the spacecraft caused by the impact; whereas for the waves, RPW measures the electric field associated with the waves. Here and elsewhere in this paper, we use antenna effective lengths from calibration by Panchenko (2017) to convert between antenna voltages and electric fields.

\section{TDS design, functionality, and performance}

The TDS instrument samples three analog signals from the highfrequency pre-amplifiers of RPW antennas $(V 1, V 2$, and $V 3)$ and one signal $\left(B_{\mathrm{MF}}\right)$ from the high-frequency winding of the search coil magnetometer (SCM, Jannet et al. 2021). The analog front end of TDS implements a bandpass filter between approximately $200 \mathrm{~Hz}$ and $350 \mathrm{kHz}$, along with a configurable gain switch that allows for an increase in the analog gain by $12 \mathrm{~dB}$, compared to the baseline low gain setting option. This gain is configured by a telecommand and can be set independently for each channel. Prior to digitization, the analog signals are routed through a multiplexer bank, which selects the signals to be digitized by each of the four analog to digital converters (ADC). For electric field measurements, the multiplexers allow us to choose between 
Table 1. Standard configurations of TDS inputs used in science operations.

\begin{tabular}{lcccc}
\hline \hline $\begin{array}{l}\text { Configuration } \\
\text { code }\end{array}$ & $\begin{array}{c}\text { Channel } \\
\mathrm{CH} 1\end{array}$ & $\begin{array}{c}\text { Channel } \\
\mathrm{CH} 2\end{array}$ & $\begin{array}{c}\text { Channel } \\
\mathrm{CH} 3\end{array}$ & $\begin{array}{c}\text { Channel } \\
\mathrm{CH} 4\end{array}$ \\
\hline SE1 & $V 1$ & $V 2$ & $V 3$ & $B_{\mathrm{MF}}$ \\
DIFF1 & $V 1-V 3$ & $V 2-V 1$ & $V 3-V 2$ & $B_{\mathrm{MF}}$ \\
XLD1 & $V 1-V 3$ & $V 2-V 1$ & $V 2$ & $B_{\mathrm{MF}}$ \\
\hline
\end{tabular}

monopole antenna measurements - where the antenna voltage relative to the spacecraft potential is measured - and dipole measurements - where differential voltages between RPW antennas are sampled instead.

The high frequency analog signals are digitized by four 14bit ADCs at a sampling frequency of $2097.1 \mathrm{ksps}$. This oversampled digital signal is afterwards decimated by a configurable factor of 4, 8, 16 or 32 after being processed by anti-aliasing FIR filters. The decimating filters are implemented in Field Programmable Gate Array (FPGA) logic, but the filter coefficients are uploaded to the FPGA by the flight software and multiple sets with slightly different characteristics can be chosen by a telecommand. This decimated waveform is then used as the input digital signal for waveform snapshots and TDS statistical products. This configurable decimation allows the TDS sampling rate to be set to one of the four values: $524.3 \mathrm{ksps}, 262.1 \mathrm{ksps}$, $131.1 \mathrm{ksps}$, or $65.5 \mathrm{ksps}$.

A large number of input configurations is allowed by the TDS input multiplexers, but only three multiplexer settings listed in Table 1 are used routinely in scientific operations. This list includes the monopole configuration $S E 1$ suitable for dust impact measurements, but the measurements contain more noise and spurious signals of spacecraft origin. The measurements in the DIFF1 dipole configuration are cleaner, but since the three RPW antennas lie in the same plane and essentially only allow for the measurement of a projection of the electric field to the antenna plane, the three dipole measurements in the same plane are redundant and this configuration does not easily allow for an analysis of variations in the voltage between the antenna and the spacecraft that is required for dust impact measurements. The best compromise is offered by the $X L D 1$, which combines two electric field dipoles in two channels and one monopole in the third channel. This configuration has been used for routine operations since August 2020, except for several special operations in SE1.

Overall, TDS supports all the operational modes of RPW (see Maksimovic et al. 2020 for discussion). Survey NORMAL and BURST modes are implemented in the same way in TDS, but BURST mode has a separate configuration that can be applied when RPW switches to the BURST mode. This typically includes higher rate products, such as the maximum amplitude (MAMP). In the selective burst modes SBM1, which are to be used during interplanetary shock crossings, and SBM2, to be used for in-situ Type III regions, TDS generates all the normal mode products, but can also generate additional periodic RSWF snapshots in SBM1 mode or additional triggered TSWF snapshots in SBM2 mode (see Sect. 3 for more details). TDS also supports the RPW backup mode in its LFM (low frequency mode) configuration, where nominal data is not generated, but TDS replaces LFR in the sampling of low frequency signals from SCM and BIAS. This mode is presently not used in flight while LFR operates nominally.

\subsection{On-board wave detection}

The TDS flight software implements on-board processing and detection of the captured snapshots which allows the instrument to classify the observations into three categories: waves, dust impacts and other signals. This algorithm described here is used to select the most relevant waveform snapshots for transmission to the ground by assigning a quality factor and also provides statistical characterization of the processed snapshots. This algorithm efficiently distinguishes between coherent waves, such as Langmuir waves, characterized by a narrow bandwidth and small peak to median ratio, and isolated voltage spikes associated with impacts of interplanetary dust, which result in a very large peak to median ratio and wide bandwidth.

In its nominal scientific operation (NORMAL, BURST or SBM1/SBM2 modes), TDS acquires one waveform snapshot every second. Each snapshot is then processed in the following steps:

(1) TDS calculates a maximum of the absolute value of all samples in the snapshot $V_{\max }$, a root mean square (rms) value, $V_{\text {rms }}$, and a median absolute value, $V_{\text {med }}$. This calculation, as well as all the subsequent steps, are only performed on one TDS channel selected by a telecommand.

(2) If $V_{\max }$ is less than a configured minimum amplitude threshold, the snapshot is discarded as insignificant and no further processing is done. Otherwise, the algorithm proceeds to the next step.

(3) A Fourier spectrum is calculated from the snapshot using the standard Welch method, by dividing the snapshot into blocks of 2048 points, applying a Hann window and a discrete Fourier transform (FFT) to each block and averaging all the FFT power spectra. Afterwards, the algorithm finds the largest peak in the spectrum (corresponding to the estimated wave frequency $f_{\text {wave }}$ ) and calculates its bandwidth $B W$ at half of the peak amplitude.

(4) A decision on event classification is made based on following conditions: if $V_{\max } / V_{\text {med }}>T_{\text {dust }}^{(1)}$ and $B W>T_{\text {dust }}^{(2)}$ the event is classified as a dust impact. Otherwise, if $V_{\max } / V_{\text {med }} \leq$ $T_{\text {wave }}^{(1)}$ and $B W \leq T_{\text {wave }}^{(2)}$ the event is a wave. Here, $T_{\text {dust }}^{(1)}, T_{\text {wave }}^{(1)}$, $T_{\text {dust }}^{(2)}$, and $T_{\text {wave }}^{(2)}$ are fixed thresholds configurable via telecommands. The criterion reflects the fact that wave observations are typically narrowband and the peak value in the snapshot is not much larger than the median value. The opposite holds for sharp transients such as the dust impacts.

(5) In the final step, the statistical values computed by TDS are updated. The wave and dust counters are incremented when wave or dust events (respectively) are identified. Maximum and rms values of snapshot amplitudes are updated based on the processed snapshot. For waves, a special processing step is added, where wave specific properties (frequency, peak, and rms amplitude) are added to a special statistics restricted to wave events. These values are used to update the values for the STAT packet defined below and the on-board collected histograms. In this step we also assign a quality factor to the current snapshot, defined nominally as $Q=V_{\max } / B W$. TDS can also use different quality factors, such as the amplitude alone.

The above procedure thus identifies the content of the waveform snapshots and assigns each wave snapshot a quality factor $Q$. The snapshots classified as waves with the highest quality $Q$ are retained in a snapshot queue in the TDS internal memory. This queue stores a configurable number of snapshots indexed by $Q$. When a new snapshot is detected and identified as a wave, the algorithm checks if the quality factor of this new snapshot exceeds the lowest $Q$ currently present in the queue. If this is the case, the snapshot with the lowest $Q$ is replaced by this new 
snapshot. If there are any empty slots in the queue, which typically happens after the queue is emptied and before enough wave snapshots are detected, the algorithm will fill in those slots with any snapshots exceeding the minimum amplitude threshold. If those snapshots are not identified as waves, they are assigned the lowest possible $Q=1$. A pre-defined number of slots in the NORMAL queue is allocated for dust snapshots - these are filled with the first dust snapshots acquired and marked as dust (no quality factor is assigned to dust impacts).

The content of the snapshot queue is periodically dumped to the RPW data processing unit (and eventually to the ground) in the form of the triggered snapshot (TSWF), which automatically empties the internal queue, making space for new data. This TSWF dump is performed by a spacecraft telecommand in a configurable interval (typically $1-6 \mathrm{~h}$ for survey mode snapshots). This period of snapshot dumps determines the data rate of the TSWF data products. The algorithm ensures that with every data dump TDS returns the best wave snapshots, a pre-defined number of dust events and if free space remains, it is filled with randomly chosen snapshots of above-noise amplitude. The regular snapshots (RSWF) are independent of this triggering mechanism and are transmitted in periodic intervals regardless of their content.

To support the operation of TDS in NORMAL and SBM2 modes, TDS implements two separate snapshot queues. One for the NORMAL (Survey) mode TSWF data and one for SBM2 mode triggered snapshots. Each queue can hold up to 6 megabytes of data (up to 48 snapshots of 16384 samples). In the SBM2 mode, the algorithm described above writes the data into those two queues in exactly the same manner - the check for the lowest $Q$ snapshot is performed for each queue separately and a sufficiently high- $Q$ snapshot will be stored in both queues. However, the SBM2 snapshot queue is dumped much more frequently, every $5 \mathrm{~min}$, providing a very high rate of TSWF snapshots. This SBM2 TSWF data is identified by a different packet ID recognized by the spacecraft data handling system which routes the SBM2 data to a dedicated data store, available for a selective download. On the other hand, the detection algorithm and the associated data products are not affected by the change to BURST or SBM1 mode. In those modes, only the cadence of periodic snapshot changes and the detection algorithm operates exactly in the same way as in NORMAL mode.

\subsection{In-flight performance}

After the launch of Solar Orbiter in February 2020, the TDS instrument has performed nominally and has been operating nearly continuously during the commissioning and during the early cruise phase, which started on June 15, 2020. During this cruise phase, which precedes the nominal mission, Solar Orbiter is to make several full orbits around the Sun scanning the heliocentric distances between $0.5 \mathrm{AU}$ and $1 \mathrm{AU}$. Already in the cruise phase TDS routinely observes all of the target phenomena examples of which are shown in Fig. 1. All the examples in Figs. 1 and 7 have been detected and correctly classified by the on-board algorithm.

Unfortunately, the performance of TDS and RPW as a whole is impacted by a strong electromagnetic interference radiated by the spacecraft which shows as strong spectral peaks at multiple frequencies in the operational range of the instrument. Figure 2 shows the background spectrum of the TDS instrument, recorded in absence of any natural signals and demonstrating all the narrowband spacecraft electric emissions seen by TDS. The displayed spectrum is from antenna $V 1$ sampled in monopole mode,
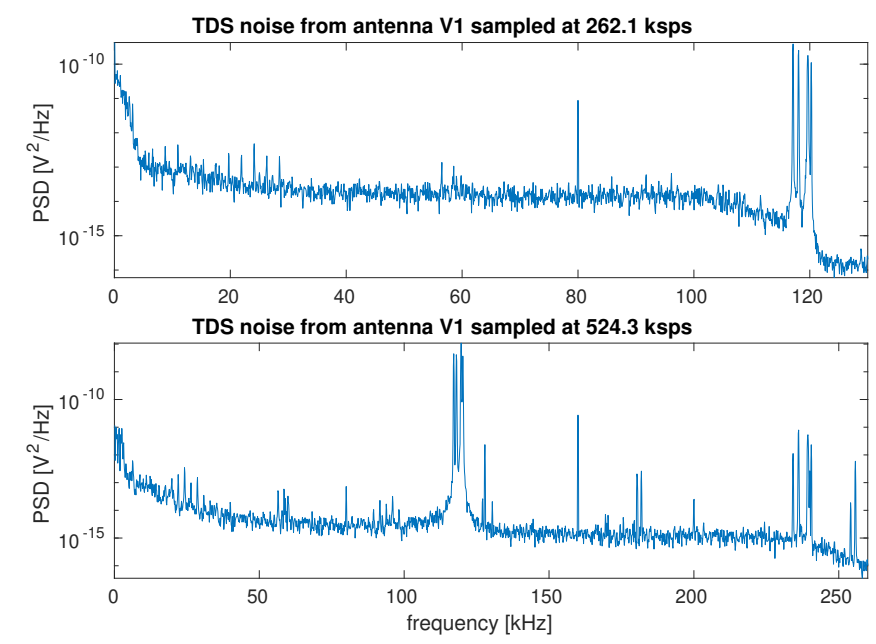

Fig. 2. Examples of power spectral density of the TDS background voltage (events with no natural signal) from antenna V1 in the lower $262.1 \mathrm{ksps}$ sampling mode (top) and in the higher $524.3 \mathrm{ksps}$ mode (bottom).

which is more susceptible to interference, and is shown separately for the higher $524.3 \mathrm{ksps}$ sampling rate mode (bottom) and lower $262.1 \mathrm{ksps}$ sampling rate mode (top). Clearly, the most significant perturbation is the cluster of spikes between 115 and $120 \mathrm{kHz}$ corresponding most likely to the emission from spacecraft power conversion and distribution unit (PCDU) radiated to space from the solar panels. Other interference peaks are much lower in amplitude and generally do not represent a major issue for the TDS measurements. The top panel shows that in the $262.1 \mathrm{ksps}$ mode, with the choice of the steepest anti-aliasing filter, this PCDU interference is already at the very edge of TDS range, attenuated by the filter, and can be relatively easily removed from the data by ground processing. In this mode, the PCDU emission does not strongly interfere with the TDS on-board detection algorithm which then performs nominally even in its presence. The situation is different for the $524.3 \mathrm{ksps}$ operation mode, where the PCDU interference falls in the center of TDS measurement range (its harmonic is also visible) and the detection algorithm falsely identifies this interference as a plasma wave. The existing implementation does not allow us to reconfigure the algorithm to work in the presence of this interference and we therefore rarely use the $524.3 \mathrm{ksps}$ mode in the first year of the mission. An update to the TDS flight software is in preparation, with the aim to mitigate this problem at least in part.

The detection algorithm has been tested and its configuration fine tuned during the commissioning phase. After April 17, 2020, a new nominal configuration has been in use, which provides a reasonably consistent capability to detect plasma waves and dust impacts in the $262.1 \mathrm{ksps}$ sampling mode. This lower sampling rate is now being used most of the time as it is adequate to the values of electron plasma frequency observed between $0.5 \mathrm{AU}$ and $1 \mathrm{AU}$. The higher sampling rate of $524.3 \mathrm{ksps}$ has been used only sparingly for special operations, because the artificial spacecraft PCDU emission discussed above interferes with TDS wave detection.

Figure 3 shows the daily counts of the detected wave and dust events from the on-board statistics. The dataset was cleaned by removing intervals when TDS was operating in a non-nominal mode, intervals of $524.3 \mathrm{ksps}$ sampling and data points acquired close to the active sweeps performed by the BIAS instrument. 


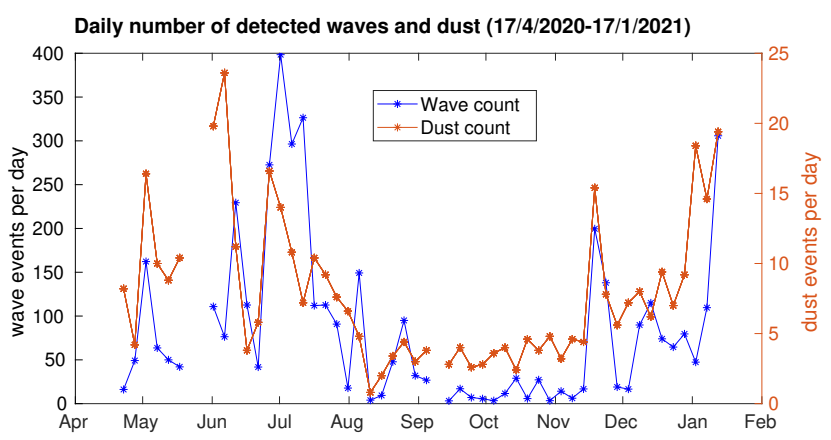

Fig. 3. Daily numbers of dust impacts and waves detected by the TDS instrument during the first nine months of the mission. We note that the vertical axis is different for waves and for dust.

We also removed the intervals around the flyby of Venus on December 27, 2020, when TDS detected numerous solitary waves, but these were incorrectly counted as dust. For a better graphical representation, the daily counts are presented as five-day averages. Clearly, the daily counts of both waves and dust impacts are modulated by the heliocentric distance with more events being detected close to the perihelion in June 2020. This dependence is of physical origin and as discussed in several studies (Píša et al. 2021; Zaslavsky et al. 2021). The high wave counts in July and in November correspond to periods of increased solar activity when TDS detected waves associated with Type III burst and other transient phenomena.

When the number of detected events is low and the TDS triggered snapshots are dumped often, most or all of the detected events included in the statistics are also transmitted as triggered snapshots. This allows to verify the correctness of event detection by TDS. Manual validation of the data shows that wave detection is very reliable and false positive wave detections are very rare except for the cases when the spacecraft interference is falsely identified as a wave. On the other hand, the algorithm is not perfect and misses some of the wave snapshots, in particular the broadband and low amplitude ones. The probability of this false negative cannot be easily established because nonselected snapshots are not transmitted to ground. The situation is somehow different in the case of dust impacts where false positives (non-dust waveforms incorrectly identified as dust) are not uncommon. Visual check of the detection efficiency in the month of September 2020 shows that 9 dust events out of the total of 73 were identified incorrectly - in particular short bursts of wave activity are sometimes misidentified as dust impacts. Furthermore, the TDS detection algorithm cannot distinguish between dust impacts and solitary waves which also present as short transient spikes in the antenna voltage. These are relatively rare in the solar wind, but very common in the magnetosphere of Venus (see Hadid et al. 2021) where TDS detected a large number of such events and falsely classified them as dust.

\section{TDS data products}

The main data product of TDS are waveform snapshots. These contain up to 65536 points per channel and up to four TDS channels can be sampled simultaneously. Furthermore, TDS generates multiple other data products, as listed below with the acronyms in parentheses:

Regular waveform snapshots (RSWF). These waveform snapshots are taken periodically at configured time intervals and their purpose is to provide un-biased duty cycled coverage of the high frequency waveform, suitable for statistical studies and detection of longer time scale phenomena, such as Type III radio bursts. During science operations, the RSWF are typically generated every 300 or $600 \mathrm{~s}$ and their length is set to 4096 or sometimes 8192 samples per channel. They are captured synchronously with the LFR snapshots and synchronized to $5 \mathrm{~min}$ boundaries to overlap with the full three-dimensional proton distributions measured by the SWA instrument. As no detection or thresholding is used for RSWF, the snapshots often contain only noise.

Triggered waveform snapshots (TSWF). The triggered waveform snapshots are a subset of waveform snapshots selected by the wave detection algorithm for transmission to the ground. These snapshots thus typically contain signals above the noise floor with a strong presence of waves and dust impacts. The snapshots are collected and stored in an internal queue over a configured time interval (typically 1 to $6 \mathrm{~h}$ ) and ranked using a quality factor assigned by the algorithm. At the end of this interval, the highest ranked snapshots are transmitted to ground. The triggered snapshots are typically configured to a length of 16384 samples per channel.

Wave, dust, and snapshot statistics (STAT). This short data packet contains several statistical quantities describing the waveform snapshots processed by the TDS detection algorithm, including those which were not selected for download as triggered snapshots. Nominally, TDS processes one waveform snapshot every second and the STAT packet transmitted every $16 \mathrm{~s}$ contains averages and counts accumulated over the past $16 \mathrm{~s}$. Included here are the number of detected waves and dust impacts, the peak and rms amplitudes of detected waves, median value of the frequencies of detected waves, and the maximum absolute value and a rms value calculated from all processed snapshots. The statistics is only calculated from one TDS channel selected by a telecommand.

Histograms (HIST1D and HIST2D). As a part of the onboard processing described in the previous section, TDS can also build on-board histograms from wave, dust and snapshot parameters calculated by the algorithm. The parameters that can be included in the statistics include peak and rms amplitude of snapshots and of identified waves, wave frequency, and the amplitude and width of identified dust spikes. It is possible to choose via telecommand up to four parameters to include in histograms and up to two pairs of parameters to be used for building of two-dimensional histograms. The cadence of the histograms is configurable, but in the typical configuration used during commissioning and cruise phase, the one dimensional histograms are transmitted every $10 \mathrm{~min}$, while the two-dimensional histograms every $30 \mathrm{~min}$.

Maximum amplitude time series (MAMP). This special product contains the maxima of an absolute value of the raw observed signals in each channel, sampled at the original $2097.1 \mathrm{ksps}$ rate. The maxima are taken over 16384 samples (equivalent to $7.8 \mathrm{~ms}$ ) or a multiple of this interval. This yields a time series of up to 128 points per second per channel. This product is always enabled in BURST mode and sometimes in NORMAL mode when telemetry is available.

There are two additional data products which are only generated in the selective burst modes of RPW (SBM1 and SBM2) and they are intended to be stored in on-board spacecraft memory for a later selective download. The SBM1 product is identical to the RSWF snapshot described above, only in the SBM1 mode, those snapshots are generated at a much higher cadence (up to 
1 snapshot per second). Analogously, in SBM2 mode, TDS provides additional triggered snapshots which are triggered in exactly the same way as the survey TSWF, only they are placed in a separate queue and can be transmitted more frequently. The only difference in TDS operation in the SBM1 and SBM2 modes is the generation of those additional data products; all other data products and operations remain exactly the same as in NORMAL mode.

\section{Statistical properties of detected Langmuir waves}

In this section, we provide an analysis of the properties of the detected waves extracted from the first year of TDS statistical data in order to demonstrate the capabilities of TDS and its onboard detection algorithm. The dataset was cleaned in the same way as for Fig. 3, but here we restricted the analysis to wave statistics. Figure 4 shows histograms of the median frequency of the waves reported in the STAT dataset, re-normalized to a number of events per day. Here the median frequency is the median value of the frequencies of all waves detected in the $16 \mathrm{~s}$ interval, so it is calculated only over the subset of snapshots (at most 16) identified as waves. The histograms are calculated separately for four intervals of heliocentric distances between $0.5 \mathrm{AU}$ and $1 \mathrm{AU}$.

The top two histograms for distances below 0.7 AU show two well separated peaks in frequency: one corresponding to ionacoustic waves at lower frequencies and the other to Langmuir waves around the electron plasma frequency which reached up to $90 \mathrm{kHz}$ at the perihelion. With increasing heliocentric distance the electron plasma frequency decreases and the Langmuir wave peak merges with the lower frequency waves. The average daily number of wave events are also shown in the histograms and they clearly show the occurrence rate of plasma waves at all frequencies increases as the spacecraft moves toward the Sun, as has also been observed for lower frequency whistler waves (Kretzschmar et al. 2021).

We attempted to separate the Langmuir waves from the ionacoustic ones by restricting our analysis to events where the median frequency was above $20 \mathrm{kHz}$. The monthly averaged daily event counts of Langmuir waves are plotted by a red line in the top panel of Fig. 5. These monthly count rates are calculated as the total number of events normalized to the total duration of available TDS measurements in that month. There are two obvious intervals of high occurrence rate of Langmuir waves, one in June and July, coincident with the perihelion, and one in November-December period, shortly after aphelion. The second interval in particular is clearly due to intense Type III activity observed clearly both in radio waves and in energetic particles on the EPD instrument (Rodríguez-Pacheco et al. 2020). However, numerous Langmuir waves were observed without any connection to Type III events or other obvious causes. These could be generated by Type III events not visible on RPW, sporadic energetic particle beams or solar wind structures such as magnetic holes or discontinuities. In order to characterize those "sporadic" events, we removed all intervals where Langmuir waves have been detected within one day from a Type III event observed in radio frequency domain by RPW.

The occurrence rate of those events is plotted with a blue line in the same panel, with the total numbers in the second panel. The occurrence rate shows a much more evident dependence on heliocentric distance then prior to Type III removal. The bottom panel shows the histograms of wave amplitudes (peak and rms) from the TDS statistics plotted separately for
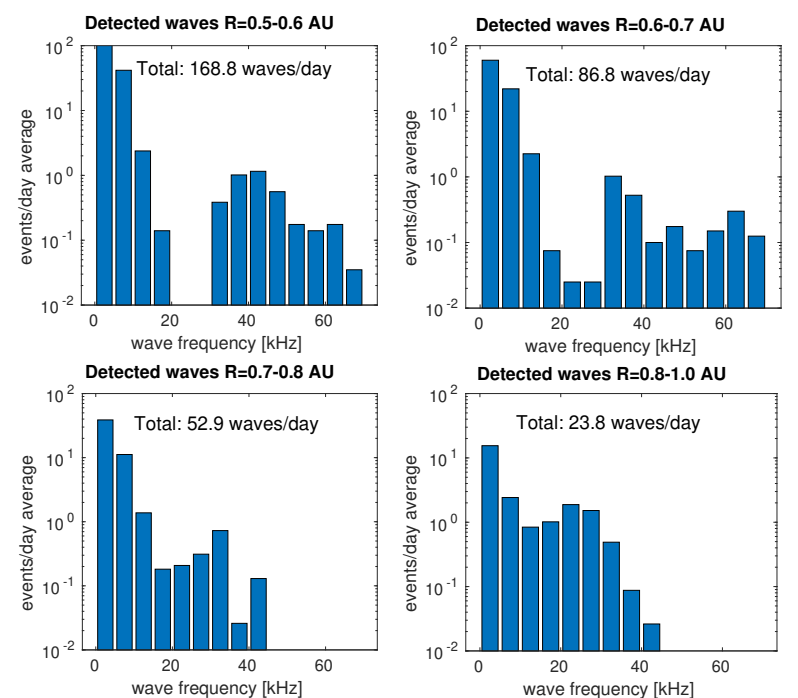

Fig. 4. Histograms of frequency of detected waves, extracted form onboard statistics for different heliocentric distances. The counts are normalized to events per day. By "waves", in this context, we mean individual TDS snapshots containing detected waves.

Langmuir waves detected close to Type III bursts (black) and the sporadic ones (blue). The histograms also clearly show that the most intense events at the tail of the distribution are mostly associated with the Type III bursts. The explanation of the observed statistical properties of the sporadic Langmuir waves is beyond the scope of this overview paper and will require a more detailed study comparing the individual observations with energetic particle observations and large-scale solar wind structures.

\section{In-situ waves associated with the November 17, 2020 Type III burst}

Solar Orbiter observed multiple Type III radio bursts in the second half of November 2020 and during some of those events, the associated energetic particles reached the spacecraft and have been detected by the EPD instrument. During those in-situ Type III observations, the RPW instrument sometimes observed the plasma waves generated by plasma instabilities triggered by those solar particles. In this section, we show an overview of the wave observations and an example of the use of TDS histogram data to characterize the wave properties.

Figure 6 provides an overview of RPW observations from November 17, 2020. The top two panels show the electric field spectrogram from the TNR receiver $(8 \mathrm{kHz}$ to $1 \mathrm{MHz}$, with the interference lines removed) and from TDS (1 to $110 \mathrm{kHz}$ ). The TDS spectrogram is calculated from the periodic RSWF waveform snapshots (with a 5 min cadence on this day) using a standard Welch FFT technique. The TNR spectrogram shows the characteristic signature of multiple type III events and for the two strongest ones that reach lower frequencies the lower frequency radio waves can be observed on TDS too. While TDS measurements have a worse time resolution limited by the configured cadence of RSWF and lower sensitivity, they offer a much higher frequency resolution and notably TDS provides a rare waveform measurement of the type III radio wave signal.

The third and fourth panels show the rms amplitudes of detected waves and their frequencies in the form of scatter plots, 

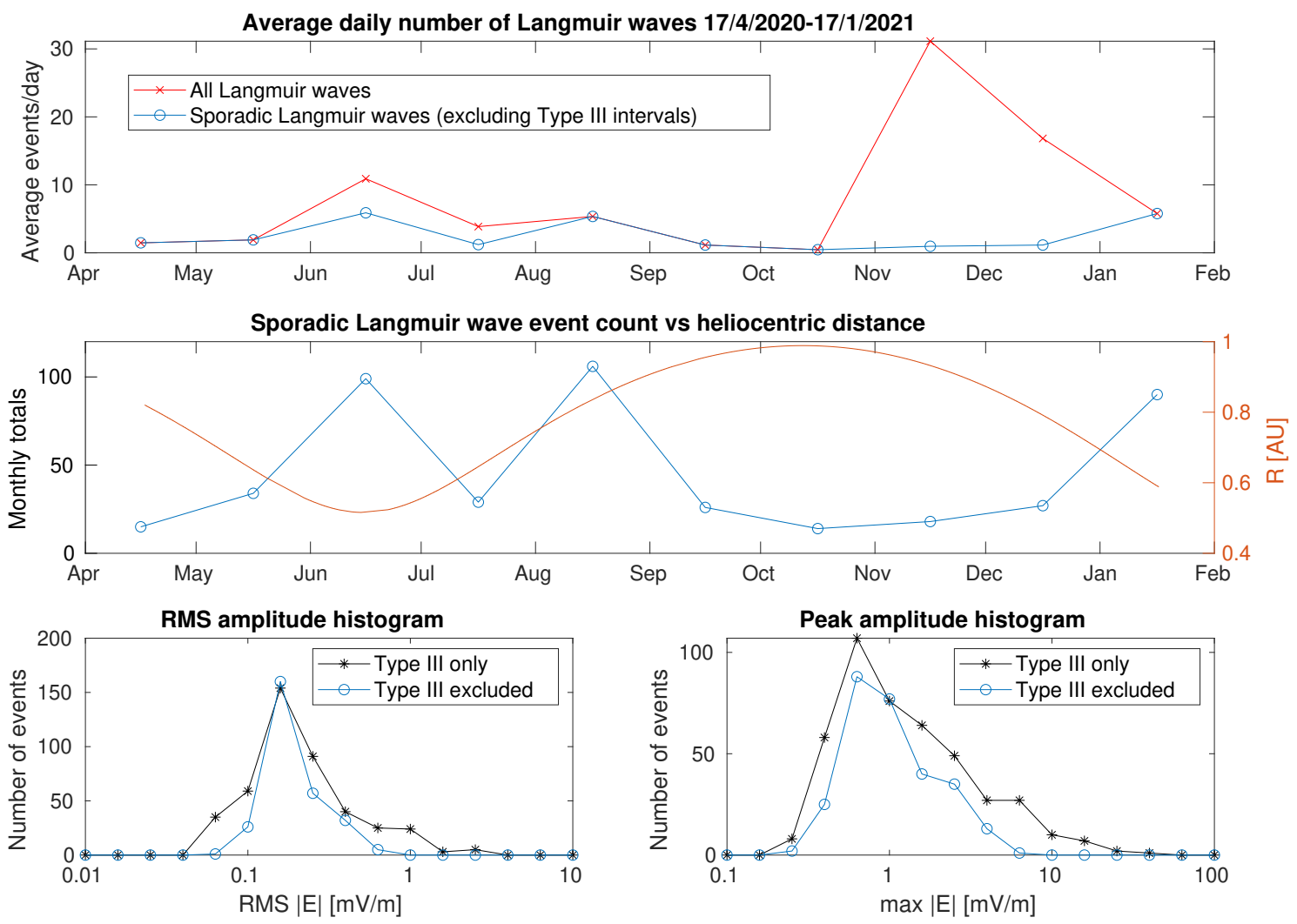

Fig. 5. Statistics of Langmuir waves detected by TDS. Top: daily number of observed Langmuir waves (monthly averages) as a function of time. The red line shows the rates for all Langmuir waves, blue line only for the waves not associated with a Type III radio burst observed by RPW ("sporadic" Langmuir waves). Middle: total monthly number of sporadic Langmuir waves with the heliocentric distance over-plotted. Bottom: histograms of peak and rms amplitudes of Langmuir waves associated with Type III events (black) and the sporadic ones (blue). By "waves", in this context, we mean individual TDS snapshots containing detected waves.

where one dot corresponds to one STAT packet containing a non-zero number of detected waves. Clearly, this compressed information matches the intervals of visible wave activity in the TNR spectrum, but provides much better resolution in timing of wave events, their amplitude and frequency. Between 3:00 and 6:00 UT on that day, RPW observed a burst of low-frequency waves possibly associated with solar energetic protons. Later in the day, numerous Langmuir waves were observed near the electron plasma frequency, which reached rather large amplitudes around the time of 14:00.

Many of these strongest Langmuir waves were selected as triggered snapshots and transmitted to ground. Figure 7 shows two examples of intense Langmuir waves from that interval. The right panels also show hodograms of the waves at the times indicated by colored stripes in the waveform plots. Since the three RPW antennas are located in the same plane, we can only display the polarization in the plane of the antennas, the $Y-Z$ plane in the spacecraft reference frame (SRF). In this coordinate system, the $V 1$ antenna is approximately parallel to the $Z$ coordinate, while the dipole $V 2-V 3$ is along the $Y$ axis (refer to Maksimovic et al. 2020 for a drawing and details). Clearly, the polarization of the wave changes between individual wave packets and many of the waves are elliptically polarized with a significant transverse component (Malaspina \& Ergun 2008; Malaspina et al. 2011), suggesting these waves are $Z$-mode waves, rather than pure electrostatic Langmuir waves (Bale et al. 1998). Despite the relatively large amplitude reaching $50 \mathrm{mV} \mathrm{m}^{-1}$, we have not yet observed the magnetic component of such waves in the TDS magnetic channel.
Figure 8 shows the averaged two-dimensional histogram of detected wave rms amplitude versus wave frequency, collected and binned by TDS on-board. This histogram is transmitted every $30 \mathrm{~min}$, but in the figure we show an averaged histogram with the counts accumulated over the entire day. The figure shows two clearly separated clusters of counts. The counts in the low frequency region below $10 \mathrm{kHz}$ correspond to the ionacoustic waves observed at the beginning of the day. The distribution of counts indicates a weak relationship between wave amplitude and frequency. The points in the higher frequency part of the histogram correspond to Langmuir waves clustered around the plasma frequency. Their distribution shows an interesting pattern where more intense waves are localized very close to the plasma frequency while the weaker waves are scattered in frequency well above and sometimes below the plasma line. This dependence appears analogous to the same pattern observed for electrostatic waves in the terrestrial foreshock (Soucek et al. 2019), where it has been shown that waves generated by faster electron beams are observed close to the plasma frequency and are more likely to correspond to Langmuir wave mode, while slower beams generate waves above and below the plasma frequency on the beam mode dispersion branch. In this case, however, the largest amplitude waves are concentrated in a very short time interval (where plasma frequency was nearly constant) and the scattered pattern is more likely due to the fluctuations of the plasma frequency during the day. Nevertheless, this example shows the value of the histogram products in particular for events with a large number of wave events and relatively reduced number of full snapshots that can be downloaded. 

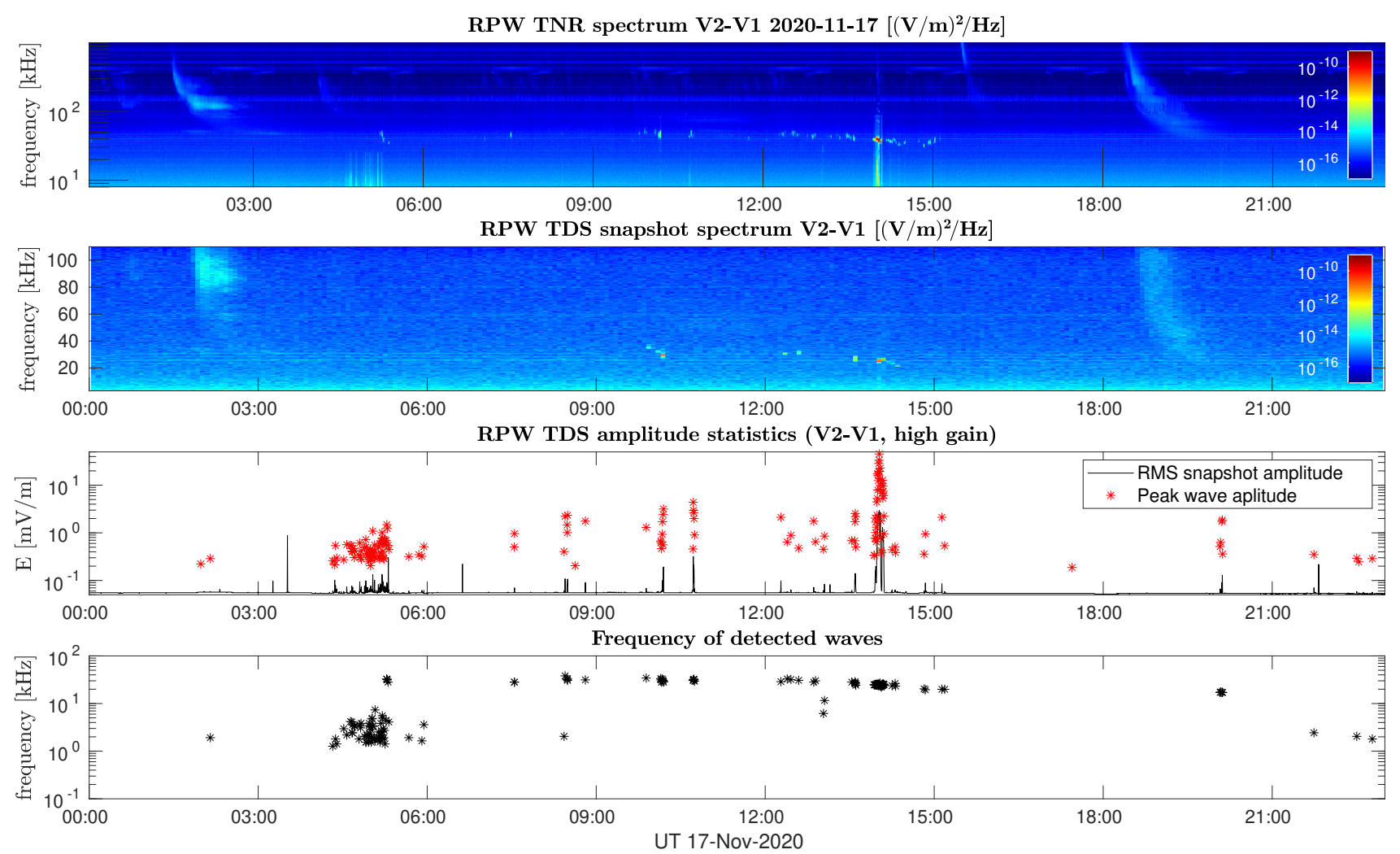

Fig. 6. Overview of RPW observations during Type III events on November 17, 2020: (a) Radio spectrum from the RPW/TNR receiver; $(b)$ Spectrogram from TDS periodic waveform snapshots; $(c)$ On-board calculated statistics of amplitudes of detected waves; $(d)$ On-board calculated statistics of frequencies of detected waves.
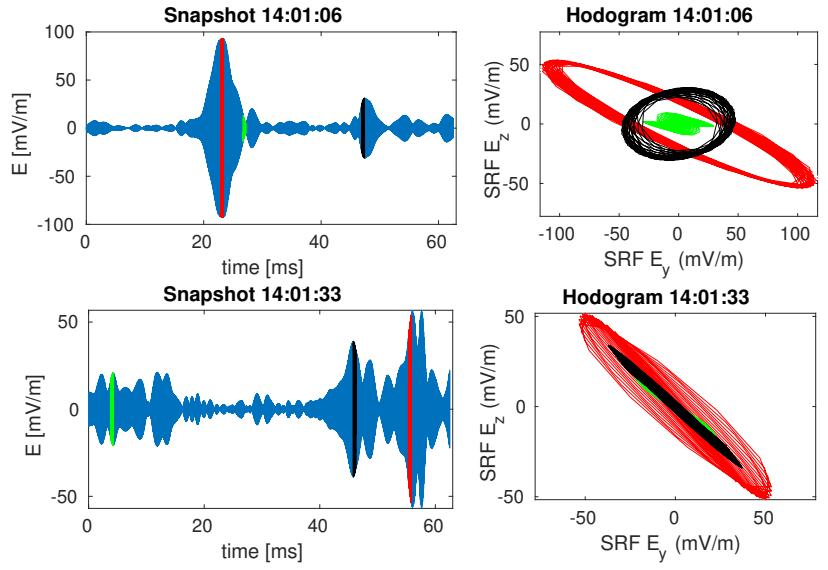

Fig. 7. Two examples of intense Langmuir waves observed during the in-situ Type III event. Hodograms in the panels on the right show wavepacket polarization in selected sections of the waveform. The hodograms are plotted in the $Y-Z$ plane of the Spacecraft Reference Frame (SRF).

\section{Conclusions}

The data from the first year of observations by the RPW-TDS instrument show that the instrument performs nominally and returns valuable scientific data even in the presence of the strong interference from the spacecraft PCDU. The TDS wave detection algorithm has been described in detail and shows a good in-flight performance in detecting plasma waves and dust impacts. The PCDU interference prevents the detection algorithm from operating at the highest sampling rate of $524.3 \mathrm{ksps}$; therefore, we

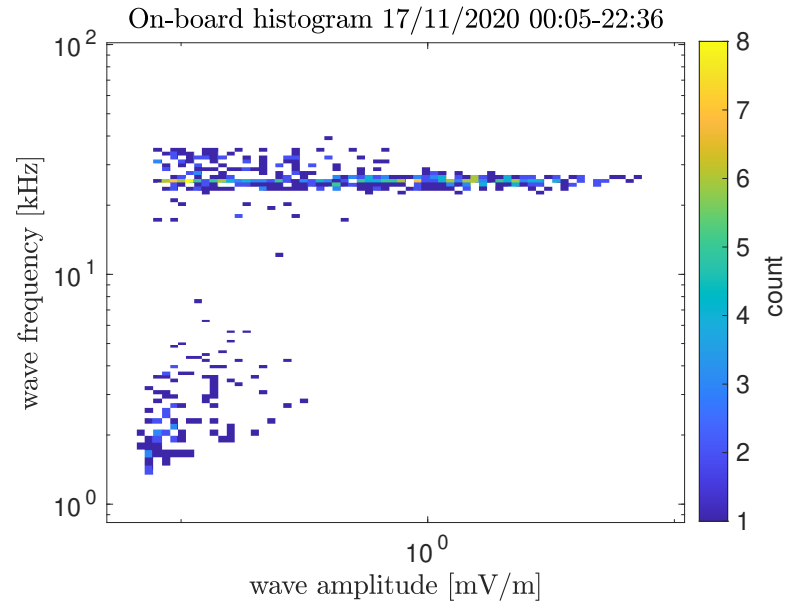

Fig. 8. Two-dimensional histogram of wave amplitude versus wave frequency collected by TDS on-board. This plot is an average of 44 individual histograms transmitted by TDS on November 17, 2020.

have been operating TDS at the $262.1 \mathrm{ksps}$ sampling throughout the cruise phase. At this sampling rate TDS can resolve frequencies up to $100 \mathrm{kHz}$, which is adequate for the cruise phase, but this issue needs to be corrected by a flight software update before Solar Orbiter ventures closer to the Sun in the nominal mission phase.

TDS was preceded by analogous instruments on previous spacecraft, notably, the Time Domain Sampler receivers on the WIND spacecraft (Bougeret et al. 1995), STEREO (Bougeret et al. 2008), and, more recently, on Parker Solar Probe 
(Bale et al. 2016), which proved valuable for studying high frequency wave processes in the solar wind (notably Langmuir waves) and also the interplanetary dust. As we have shown in this article, the Solar Orbiter RPW TDS offers comparable performance in the observation of the phenomena and offers some new aspects and features, in particular in terms of wave and dust detection and on-board statistics. All the instruments mentioned above implement some triggering to detect relevant sections of waveform. While the earlier instruments generally only selected the highest amplitude events, the FIELDS instrument on Parker Solar Probe allows for more varied detection techniques, taking into account an estimate of wave frequency based on the number of zero crossings. In comparison, Solar Orbiter TDS uses a slightly different and arguably more complex algorithm, allowing it to detect a wider variety of events, in particular weaker waves and less intense dust impact spikes. This, combined with its rich statistical products, makes RPW/TDS suitable for both detailed measurements and statistical surveys of high frequency phenomena in the inner heliosphere.

An initial analysis of the TDS statistical data products shows that Langmuir waves are sporadically observed outside identified Type III regions with a clear increase in occurrence rate with decreasing distance to the Sun. The amplitude of those waves is typically lower than those associated with Type III events. While the waves associated with Type II and type III events have been studied extensively, including observations from the inner heliosphere (Gurnett \& Anderson 1977), these more sporadic events have not been specifically addressed in previous studies. A recent study by Graham et al. (2021) suggests that an increased occurrence of such sporadic Langmuir waves is associated with solar wind current structures. A subsequent and more detailed analysis of those sporadic waves is needed to understand their origin.

Overall, RPW has observed numerous Type III burst events (e.g., Gómez-Herrero et al. 2021). During a series of Type III radio bursts on November 17, 2020, TDS captured a large number of intense Langmuir waves when Solar Orbiter passed through the source region of the radio emission. We demonstrate here that the on-board calculated histograms from TDS allow us to classify wave observations and identify physically relevant correlations between individual wave parameters. Multicomponent waveform measurements of TDS allow us to resolve the polarization and modulation of the waves, identifying numerous waves with a strong elliptical polarization.

Acknowledgements. Solar Orbiter is a space mission of international collaboration between ESA and NASA, operated by ESA. RPW-TDS development has been developed with the support from the Czech PRODEX program funded by the Czech Ministry of Education, Youth and Sports. The TDS team thanks the scientific and engineering teams or other RPW subsystems as well as the team from the French Space Agency CNES for their support of the TDS development. YK is supported by the Swedish National Space Agency grant 20/136. All Solar Orbiter data is available from the public Solar Orbiter Archive (http: // soar.esac.esa.int).

\section{References}

Bale, S. D., Kellogg, P. J., Goetz, K., \& Monson, S. J. 1998, Geophys. Res. Lett., 25, 9

Bale, S. D., Reiner, M. J., Bougeret, J. L., et al. 1999, Geophys. Res. Lett., 26, 1573

Bale, S. D., Goetz, K., Harvey, P. R., et al. 2016, Space Sci. Rev., 204, 49

Bougeret, J. L., Kaiser, M. L., Kellogg, P. J., et al. 1995, Space Sci. Rev., 71, 231

Bougeret, J. L., Goetz, K., Kaiser, M. L., et al. 2008, Space Sci. Rev., 136, 487

Briand, C., Soucek, J., Henri, P., \& Mangeney, A. 2010, J. Geophys. Res.: Space Phys., 115, A12113

Cairns, I. H., \& Melrose, D. B. 1985, J. Geophys. Res., 90, 6637

Gary, S. P. 1985, J. Geophys. Res., 90, 8213

Ginzburg, V. L., \& Zhelezniakov, V. V. 1958, AZh, 35, 694

Gómez-Herrero, R., Pacheco, D., Kollhoff, A., et al. 2021, A\&A, 656, L3 (SO Cruise Phase SI)

Graham, D. B., \& Cairns, I. H. 2015, J. Geophys. Res.: Space Phys., 120, 4126

Graham, D. B., Khotyaintsev, Yu. V., Vaivads, A., et al. 2021, A\&A, 656, A23 (SO Cruise Phase SI)

Gurnett, D. A. 1991, Waves and Instabilities in Physics of the Inner Heliosphere II: Particles, Waves and Turbulence (Springer), 135

Gurnett, D. A., \& Anderson, R. R. 1976, Science, 194, 1159

Gurnett, D. A., \& Anderson, R. R. 1977, J. Geophys. Res., 82, 632

Hadid, L. Z., Edberg, N. J. T., Chust, T., et al. 2021, A\&A, 656, A18 (SO Cruise Phase SI)

Jannet, G., Dudok de Wit, T., Krasnoselskikh, V., et al. 2021, J. Geophys. Res.: Space Phys., 126, e2020JA028543

Kellogg, P. J. 1980, ApJ, 236, 696

Kim, E.-H., Cairns, I. H., \& Robinson, P. A. 2007, Phys. Rev. Lett., 99, 015003

Kretzschmar, M., Chust, T., Krasnoslekikh, V., et al. 2021, A\&A, 656, A24 (SO Cruise Phase SI)

Lin, R. P., Potter, D. W., Gurnett, D. A., \& Scarf, F. L. 1981, ApJ, 251, 364

Lin, N., Kellogg, P. J., MacDowall, R. J., Tsurutani, B. T., \& Ho, C. M. 1996, A\&A, 316, 425

Maksimovic, M., Bale, S. D., Chust, T., et al. 2020, A\&A, 642, A12

Malaspina, D. M., \& Ergun, R. E. 2008, J. Geophys. Res., 113, A12108

Malaspina, D. M., Cairns, I. H., \& Ergun, R. E. 2011, Geophys. Res. Lett., 38, L13101

Meyer-Vernet, N., Maksimovic, M., Czechowski, A., et al. 2009, Sol. Phys., 256, 463

Müller, D., St. Cyr, O. C., Zouganelis, I., et al. 2020, A\&A, 642, A1

Píša, D., Souček, J., Santolík, O., et al. 2021, A\&A, 656, A14 (SO Cruise Phase SI)

Panchenko, M. 2017, Scientific Report for FFG/ASAP 11 Project SOLOCAL, 847978

Rodríguez-Pacheco, J., Wimmer-Schweingruber, R. F., Mason, G. M., et al. 2020, A\&A, 642, A7

Soucek, J., Píša, D., \& Santolík, O. 2019, J. Geophys. Res.: Space Phys., 124, 2380

Zaslavsky, A., Mann, I., Soucek, J., et al. 2021, A\&A, 656, A30 (SO Cruise Phase SI) 